\title{
OBSERVATIONS OF MAGNETIC FIELD CHANGES \\ IN ACTIVE REGIONS
}

\author{
K. L. HAR VEY \\ Lockheed Solar Observatory, Saugus, Calif., U S.A. \\ and \\ W. C. LIVINGSTON, J. W. HARVEY, and C. D. SLAUGHTER \\ Kitt Peak National Observatory*, Tucson, Arizona, U.S.A.
}

\begin{abstract}
A time sequence of longitudinal magnetograms of two active regions, McMath Regions 9281 and 9760 , have indicated magnetic field changes occurring in localized areas with time scales of the order of hours. We believe the observed field changes are evolutionary in nature, rather than related to the occurrence of small flares. Three examples of evolutionary magnetic changes are discussed.
\end{abstract}

During the past two years, Kitt Peak National Observatory and the Lockheed Solar Observatory have participated in a cooperative program to observe simultaneously the magnetic fields and $\mathrm{H} \alpha$ activity in four active regions. The main purpose of this program was to attempt to observe magnetic field changes using high-time resolution magnetograms. This paper is a brief report of the analysis of the observations of two regions, McMath Regions 9281 and 9760.

At Kitt Peak, a time sequence of longitudinal magnetograms was made using a Babcock-type magnetograph (Livingston, 1968) at a rate of one magnetogram per 80 and 120 s for McMath Regions 9281 and 9760 , respectively. Spatial resolution of the magnetic observations was no better than 2.5 arc s (size of the entrance aperture). During the study of these two regions, approximately 800 magnetograms were made over a total of $20 \mathrm{hr}$ observing time. For most of the duration of the magnetic observations at Kitt Peak, simultaneous center $\mathrm{H} \alpha$ or off-band $\mathrm{H} \alpha$ filtergrams were obtained by the Lockheed Solar Observatory.

The longitudinal magnetograms of McMath Regions 9281 and 9760 have shown field changes occurring in localized areas having a time scale of the order of hours; Ribes (1969) has found similar time scales for field changes in active regions. We could infer no relation between the field changes and the 55 flares observed in Region 9760, though no flare of importance greater than 1 was observed; this confirms the results of an earlier study by Godovnikov et al. (1964). Most of the observed magnetic field changes occurred outside the areas which flared. Although no flares were observed in Mc Math Region 9281, a filament eruption occurred during the course of an underlying field change.

Our observations of magnetic field changes suggest that the field changes we

* Operated by the Association of Universities for Research in Astronomy, Inc., under contract with the National Science Foundation. 
observed are evolutionary in nature, rather than being associated with $\mathrm{H} \alpha$ flare activity. Three examples of this behavior are as follows:

(1) Observations of McMath Region 9281 began on 21 March 1968 approximately $13 \mathrm{hr}$ after the birth of the region and continued through $24 \mathrm{March}$. As shown in Figure 1, the magnetic polarities were initially aligned north-south; by 22 March the region had developed to a more nearly east-west orientation, which it retained. Weart and Zirin (1969) and Weart (1970) have also noted some new regions emerging with high inclinations and developing toward an east-west orientation.

The change in orientation of Region 9281 appears to be due to the emergence of new flux within the boundaries of the region, rather than a horizontal movement of

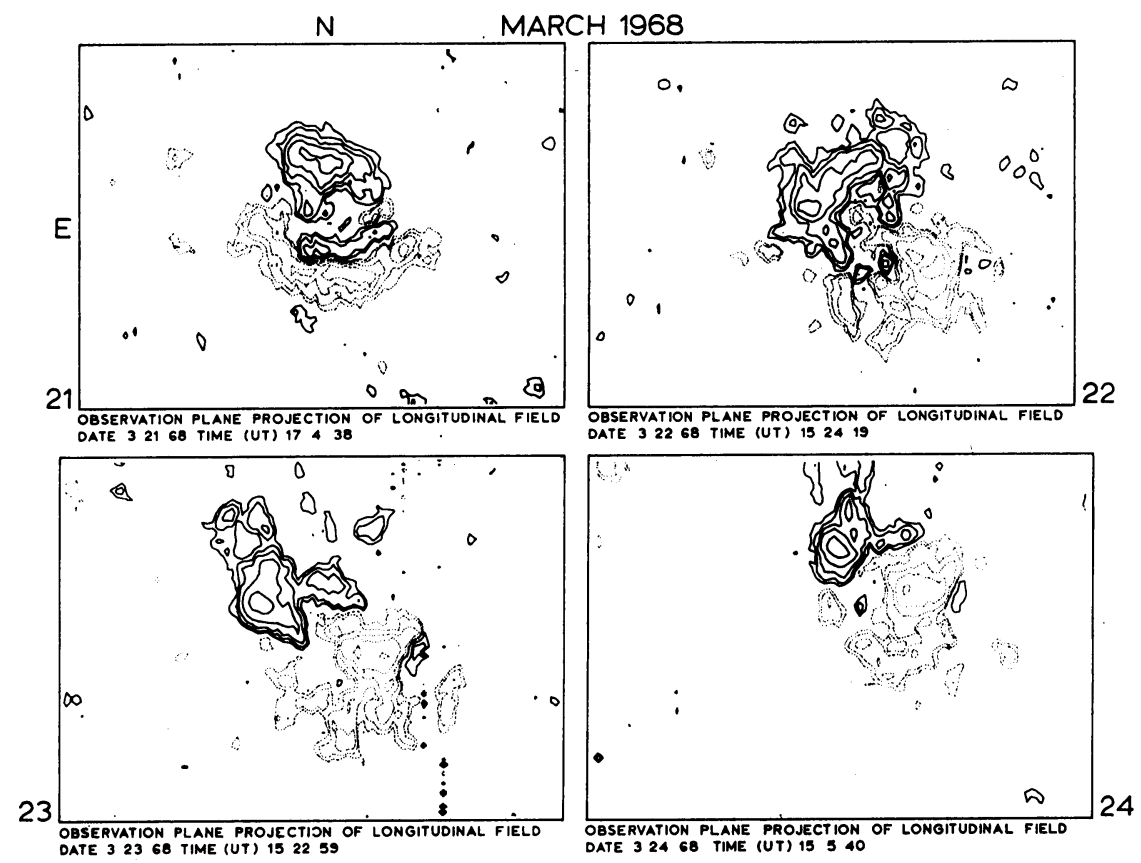

Fig. 1. Isogauss contours of the longitudinal magnetic field of McMath Region 9281 for 21 through 24 March 1968. The contour levels are 100, 200, 400, 800, and $1600 \mathrm{G}$.

the established flux. On 21 March 1968, as shown in Figure 2, two significant field changes, occurring over a period of several hours, were observed: (1) a strengthening of the positive field in an area of negative polarity along the eastern border of the region, and (2) a strengthening of negative fields along the western border of the region. In both cases, the field changes preceded and accompanied the growth of sunspots, which developed into the principal spots of the region on 22 March 1968, and thus established the more east-west orientation of the region. The birth of sunspots in areas of increasing field strength has been observed by Gopasyuk (1967) and Ogir and Shaposhnikova (1965). 
(2) Our observations suggest that McMath Region 9281 emerged with a definite non-potential field configuration and evolved to a more potential-like configuration. In Figure 3, we have compared the orientation of the $\mathrm{H} \alpha$ fine structure with the computed transverse component of a potential field configuration calculated using the observed longitudinal field distribution. The $\mathrm{H} \alpha$ fine structure is assumed to be aligned along field lines as indicated by the work of Bruzek (1969) and Tsap (1965).

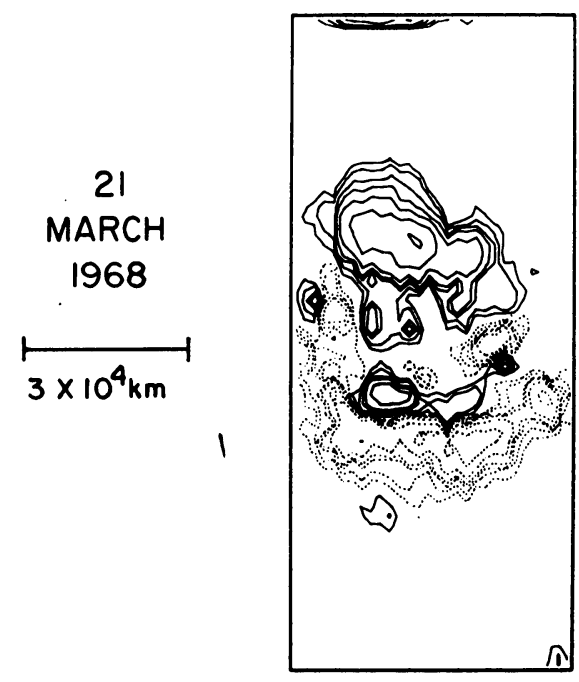

183137 UT

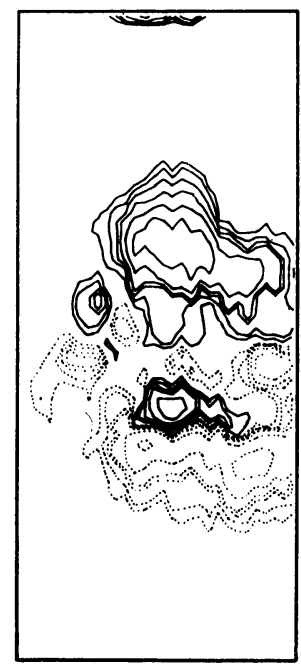

$20 \quad 0 \quad 10$ UT

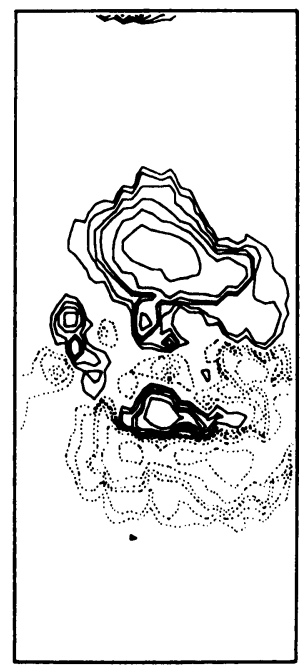

$2030 \quad 6$ UT

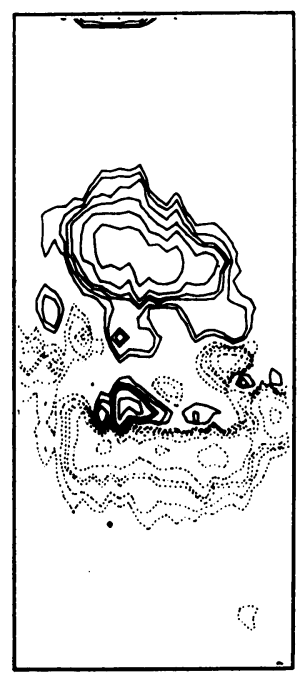

19019 UT

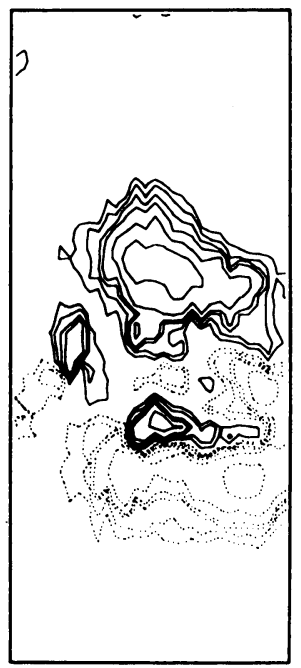

$21 \quad 132$ UT

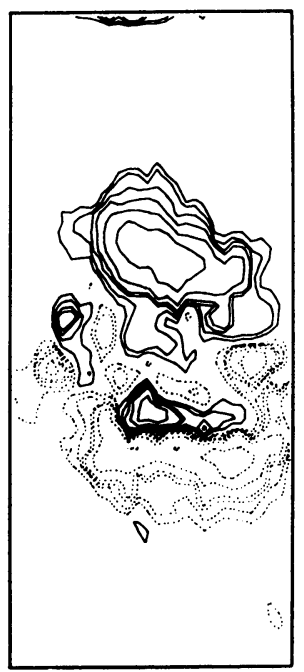

193017 UT

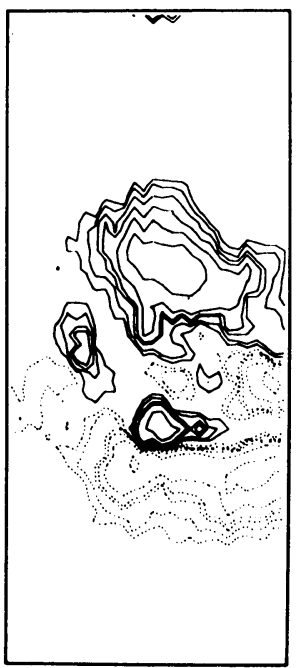

212756 UT

7ig. 2. Isogauss contours of the longitudinal magnetic field shown at roughly one-half hour intervals on 21 March 1968 of Mc Math Region 9281. 
On $21 \mathrm{March}$, the $\mathrm{H} \alpha$ inter-region fine structure showed poor correspondence with the calculated transverse field. The alignment improves by $22 \mathrm{March}$ and is quite good on 23 March 1968 (not shown in Figure 3) when the region has reached its maximum development.

(3) Our observations of McMath Region 9760 indicate a change in the direction of

\section{$21 \quad M A R C H \quad 1968$}

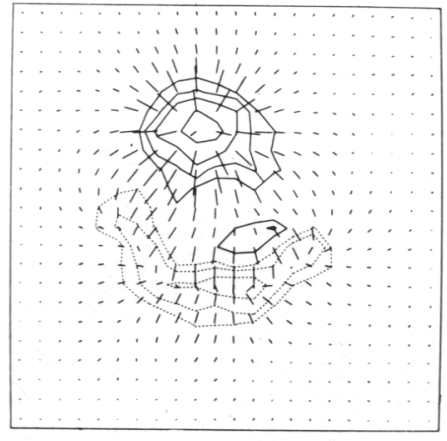

$1704 \mathrm{UT}$

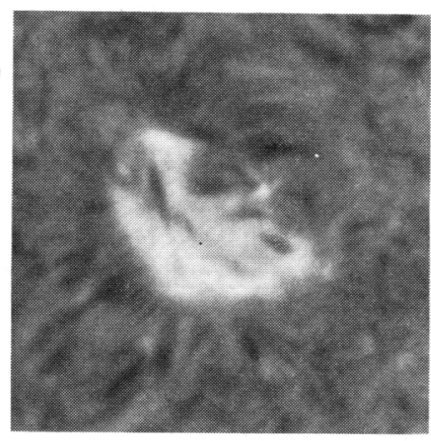

$1650 \mathrm{UT}$

\section{MARCH 1968}

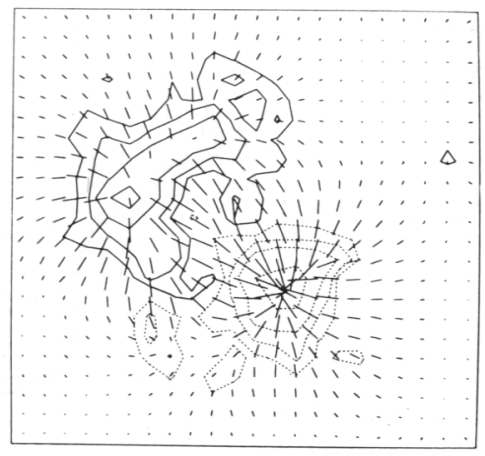

$1524 \cup T$

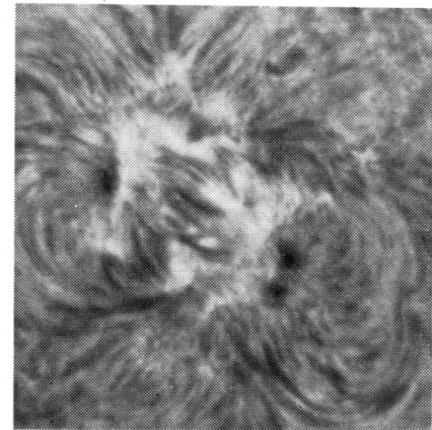

$$
2000 \text { UT. }
$$

Fig. 3. Comparison of the transverse component of a potential field (calculated from the observed longitudinal field distribution) with $\mathrm{H} \alpha$ fibril structures in Region 9281.

the transverse field from 8 to 11 November 1968 in the vicinity of the longitudinal neutral line, the line of polarity reversal. The observed transverse field is shown in Figure 4 for $8,9,10$ and 11 November. The orientation of the lines indicates the direction of the transverse field and the dark line represents the position of the polarity reversal line with respect the transverse field. On November 8 , the direction of the transverse field is inclined to the neutral line. On successive days, the inclination de- 
creased and by November 11 , the transverse field is parallel with the neutral line. Similar alignments of the transverse field with the $\mathbf{H}_{\|}=0$ line have been noted by Moreton and Severny (1968) and Rayrole and Semel (1970). The $\mathrm{H} \alpha$ fibril structures in the area of the neutral line also showed a similar behavior.

Because of the many problems in observing and interpreting measures of the transverse field, it is difficult to be certain that the observed change in the transverse field direction is entirely real.

In addition to the longitudinal magnetic field observations discussed above, a time

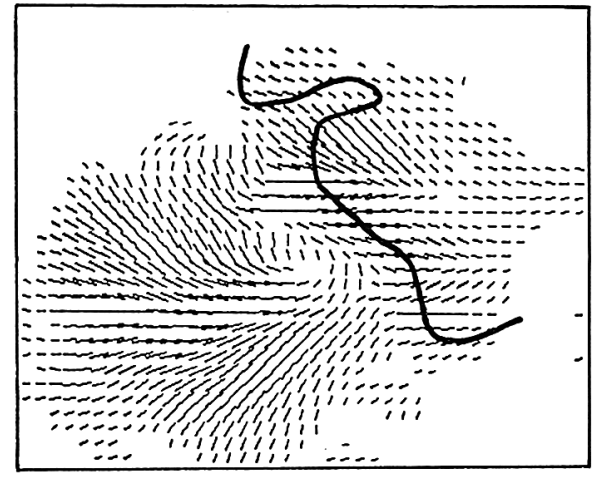

8 NOVEMBER 1968

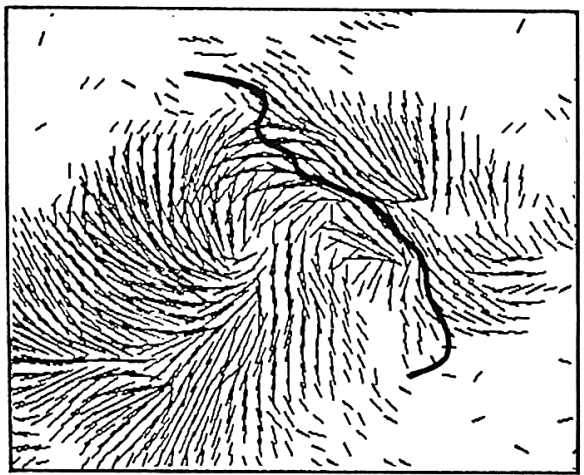

IO NOVEMBER

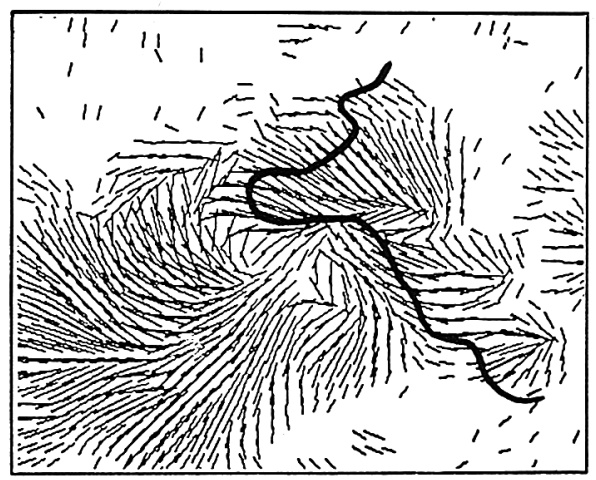

9 NOVEMBER

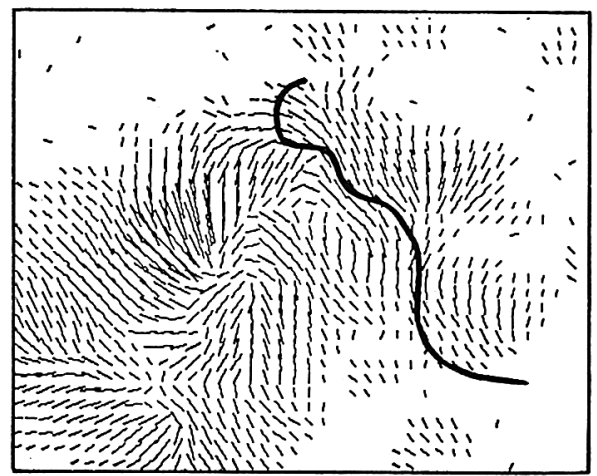

II NOVEMBER

Fig. 4. Position of the polarity reversal line (dark line) relative to the observed transverse field.

sequence (12 magnetograms per hour) of (1) the transverse and longitudinal magnetic fields of McMath Region 10148 and (2) the longitudinal magnetic fields measured simultaneously in $\mathrm{H} \alpha$ and Fe $\mathrm{I} 6569 \AA$ of McMath Region 10385 have been made.

The results of these observations and, separately, a more detailed report of the ongitudinal magnetic field observations will be published in the future. 


\section{References}

Bruzek, A.: 1969, Solar Phys. 8, 29.

Godovnikov, N. V., Ogir, M. B., and Shaposhnikova, E. F.: 1964, Izv. Krymsk. Astrofiz. Obs. 31, 216. Gopasyuk, S. I.: 1967, Izv. Krymsk. Astrofiz. Obs. 36, 56.

Livingston, W. C.: 1968, Appl. Opt. 7, 425.

Moreton, G. E. and Severny, A.: 1968, Solar Phys. 3, 282.

Ogir, M. B. and Shaposhnikova, E. F.: 1965, Izv. Krymsk. Astrofiz. Obs. 33, 92.

Rayrole, J. and Semel, M.: 1970, Astron. Astrophys. 6, 288.

Ribes, E.: 1969, Astron. Astrophys. $2,316$.

Severny, A.: 1969, in Annals of the ISQY, MIT Press, Cambridge, Mass., 3, 11.

Tsap, T. T.: 1965, Izv. Krymsk. Astrofiz. Obs. 33, 92.

Weart, S. R.: 1970, Astrophys. J. 162, 987.

Weart, S. R. and Zirin, H.: 1969, Publ. Astron. Soc. Pacific 81, 270.

\section{Discussion}

Michard: The kind of changes of magnetic patterns that you showed, with one pole increasing and another (of opposite polarity) decreasing, are consistent with the description by the Meudon observers (Martres et al.). But do you agree with their finding that $\mathrm{H} \alpha$ brightenings in flares are located on these features evolving in opposing directions?

Harvey, $K .:$ No, not for the regions we observed.

Meyer: You mentioned that the evolution of the active region considered in your first slides shows a tendency to approach a potential field configuration. Does this also hold for the increasing alignment of the tangential field component with the neutral line of the vertical flux distribution shown in your last slide?

Harvey, $K .:$ No. 